\title{
Full-Field Hard X-Ray Microscope Designed for Materials Science Applications
}

Imke Greving $^{1}$, Silja Flenner ${ }^{1}$, Emanuel Larsson ${ }^{1}$, Malte Storm $^{2}$, Fabian Wilde $^{1}$, Erica Lilleodden ${ }^{1}$, Thomas Dose $^{1}$, Hilmar Burmester ${ }^{1}$, Lars Lottermoser ${ }^{1}$, Christian David ${ }^{3}$ and Felix Beckmann ${ }^{1}$

1. Institute of Materials Research, Helmholtz Zentrum Geesthacht, Geesthacht, Germany.

2. Diamond Light Source, Didcot, UK.

3. Paul Scherrer Institue, Villigen, Switzerland.

* Imke Greving, imke.greving@hzg.

One major challenge in the research and development of new materials is their structural and mechanical characterization down to the nanometer scale. For the development of new materials with outstanding capabilities it is often essential to monitor the structural properties of the material in each step of the production process on a representative sample volume in a non-destructive way. In particular when it comes to in situ experiments at high spatial resolutions, where sufficient volumetric data are desirable, the number of suitable techniques becomes very limited. One technique well suited for this application is full-field hard X-ray microscopy (XRM).

The nano branch of the P05 imaging beamline [1] operated by the Helmholtz Zentrum Geesthacht, located at the PETRA III storage ring at DESY (Hamburg, Germany) is specifically optimized for material sciences applications [2]. The high flexibility of the setup allows switching between X-ray microscopy and cone beam configuration using compound refractive lenses. Currently the setup is equipped with an X-ray microscope offering a large focal distance at energies ranging from $10-14 \mathrm{keV}$ with an option for Zernike phase contrast. A layout of the imaging beamline as well as an image of the experimental setup is shown in figure 1 and 2.

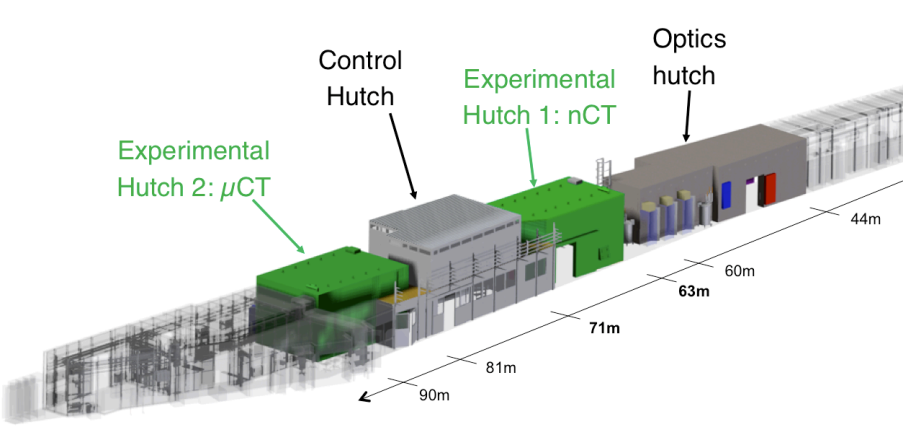

Figure 1. Overview image of the Imaging Beamline IBL / P05 at the PETRA III storage ring. The XRM is situated in the first experimental hutch (EH1), at 63 - $71 \mathrm{~m}$ from the source. A unique sample to detector distance of $18-22 \mathrm{~m}$ can be realized by placing the detector in the $2^{\text {nd }}$ experimental hutch (EH2).

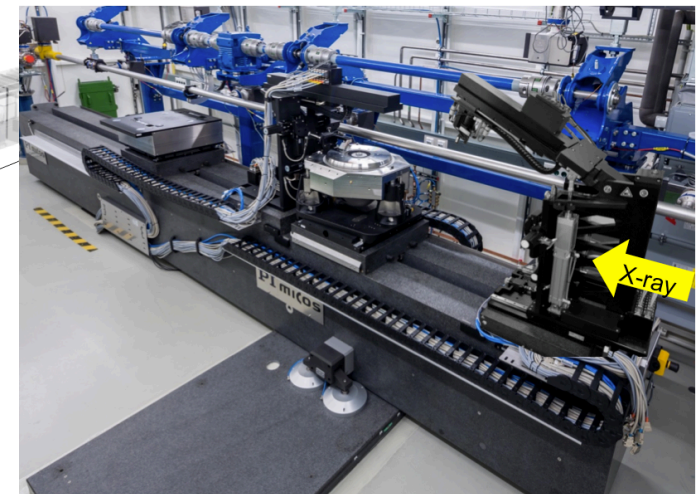

Figure 2. Photo of the experimental setup of the P05 nanotomography station. The $6.8 \mathrm{~m}$ long granite serves as an optical bench, equipped with 4 granite sliders. The blue structure in the back is a robot for moving the vacuum pipe in and out, switching between micro- and nanotomography stations $\mathrm{EH} 1$ and $\mathrm{EH} 2$. 


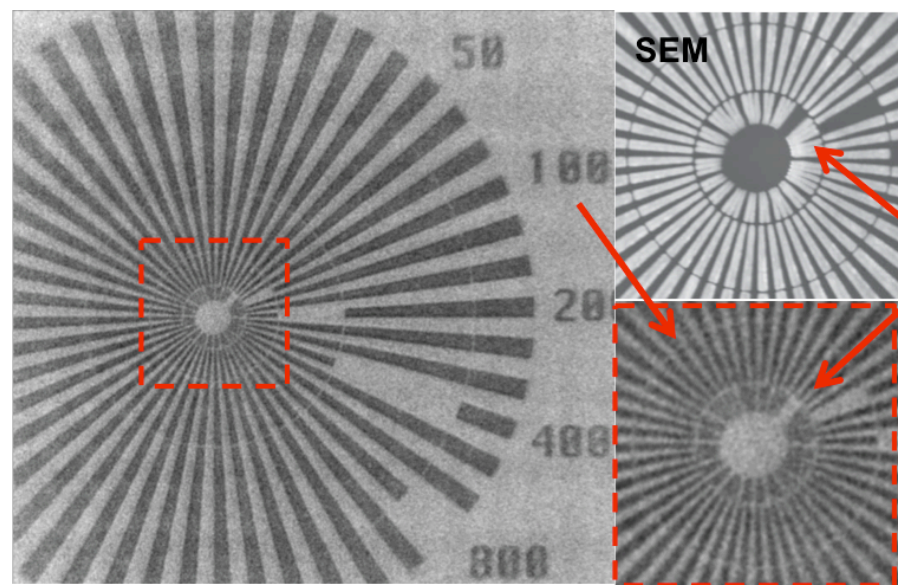

Figure 3. Siemens star acquired by XRM at $11 \mathrm{keV}, 100 \mathrm{~nm}$ lines well resolved. The inner ring (lower right close up) suggests that the $50 \mathrm{~nm}$ lines of the test pattern are collapsed. This is confirmed by an SEM image as indicated by the arrows (close up upper right).

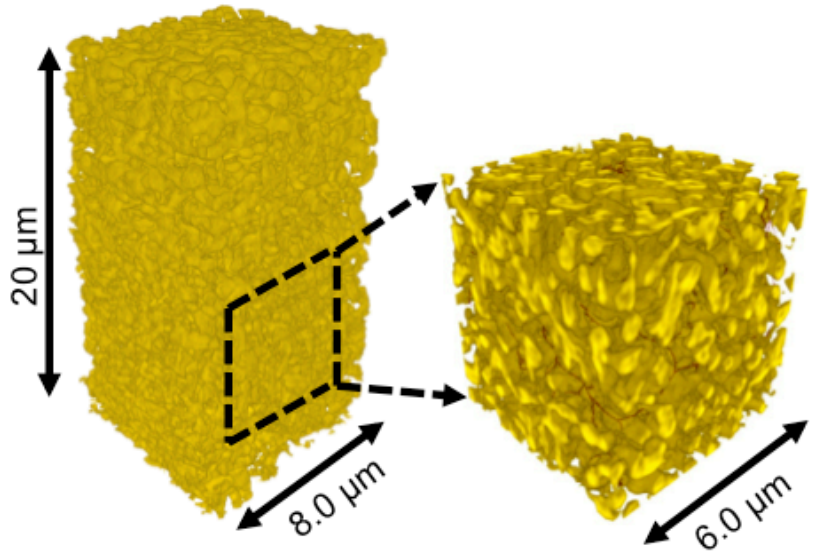

Figure 4. Volume rendering of a nano porous gold sample (NPG). In contrast to other techniques XRM allows scanning of representative volumes within $1-2 \mathrm{~h}$. This allows quantitative analysis of several sub volumes using advanced software tools e.g. Pore3d [4].

The Fresnel Zone Plates (FZP) as well as the illumination optics used for this XRM setup are designed and fabricated at the Paul Scherrer Institute. In the standard XRM configuration these optics allow for an effective pixel size of $15-20 \mathrm{~nm}$ depending on the chosen energy resulting in a spatial resolution of $100 \mathrm{~nm}$ (see Fig.3). A volume rendering of a nano porous gold sample (NPG) is shown in Figure 4. The strength of this material produced by electrochemical dealloying is determined by the mean ligament sizes. Studying this material using Focused Ion Beam tomography requires the infiltration of the NPG with epoxy [5] and is of course a destructive technique. Therefore XRM was chosen to study the structural details of NPG, allowing to scan representative volumes of non-infiltrated samples. The acquired tomographic scans using the XRM here presented allowed for quantitative determination of ligament sizes, volume fractions, pore sizes etc. using the Pore3D software package [4]. In addition to the existing microscope-type detector setup (PCO camera, $100 \mu \mathrm{m} \mathrm{CdWO} 4$ scintillator, Nikon objective) a Hamamatsu detector (C12849-101U) has been commissioned. Here no light-optical system is needed since the Gadox scintillator is directly grown on a fibre optics plate, resulting in a high photon efficiency. Placing the detector at $18.8 \mathrm{~m}$ behind the sample allows filling the whole detector chip and reducing the time for a full tomographic XRM scan to $1-2 \mathrm{~h}$ limited by the photon flux.

In the future this scan time is going to be decreased even further by commissioning of the new multilayer monochromator [1] working towards in situ and time resolved experiments at the nano scale. Overall the nanotomography station of the imaging beamline IBL/P05 is well suited for materials research currently offering XRM and Zernike phase contrast techniques.

\section{References:}

[1] F. Wilde et al. AIP Conf. Ser., vol. 1741 (2016), pp. (030035) 1-4

[2] I. Greving et al. J. of Phys.: Conf. Ser. 849 (2017), p. 012056.

[3] D. Gürsoy et al. Scientific Reports vol. 7, (2017), p. 11818

[4] F. Brun et al. Nucl. Instr. M. Phys. Res. Sect. Acce. Sp. Det. Assoc. Equ., vol. 615 (2010) p. 326-332

[5] K. Hu et al. Philosophical Magazine, vol. 96 (2016), p. 3322-3335

[6] The authors I.G., E.L. and S.F. acknowledge financial support from the German Research

Foundation (DFG) via the SFB 986-M3, projects B2 and Z2. 